\title{
M. Tiedge \\ Beta cell regeneration meets autoimmunity: PAX4 variants in type 1 diabetes
}

Published online: 19 April 2005

C) Springer-Verlag 2005

Type 1 diabetes may be considered as a disease that results from an imbalance between selective autoimmune destruction of pancreatic beta cells and beta cell regeneration. This gives rise to an intriguing question: is impaired beta cell regeneration in itself an important determinant of susceptibility to type 1 diabetes [1,2]? Two lines of investigation have recently - and more or less independently - investigated the mechanisms that underlie beta cell development and regeneration, and the mechanisms involved in beta cell destruction by immune cells and proinflammatory cytokines. In this issue of Diabetologia, Biason-Lauber and coworkers provide evidence that a genomic variant of PAX4 is linked to the development of type 1 diabetes [3].

PAX4 is a member of the Pax family of homeodomain transcription factors that are operative during the development of the endocrine pancreas and have been shown to be essential for beta cell development and beta cell function [4]. In mice, this transcription factor is expressed on or around Day 9 in cells of the dorsal pancreatic rudiment, with peak expression occurring between Day 13 and 15, followed by a decline until birth. PAX4 has been identified as a downstream target of neurogenin 3, another important transcription factor for the development of the endocrine pancreas [4]. Studies on pax4 knock-out mice showed a profound reduction in beta cell mass and a severe diabetic syndrome after birth. Atypically clustered alpha cells and ghrelin-positive cells were detected in the pancreas, and it was concluded that PAX4 suppresses the development of these cell types [4].

The pivotal role played by PAX4 in beta cell development and function has prompted genetic linkage analyses of this gene in cohorts of diabetic patients, with particular focus on type 2 diabetes. These studies yielded conflicting

\footnotetext{
M. Tiedge $(\square)$

Institute of Medical Biochemistry and Molecular Biology,

Faculty of Medicine, University of Rostock,

Schillingallee 70 ,

18057 Rostock, Germany

e-mail: markus.tiedge@med.uni-rostock.de

Tel.: +49-381-4945751

Fax: $+49-381-4945752$
}

results. Screening of French MODY families showed no linkage between type 2 diabetes and markers of PAX4 [5]. Although the $P 321 H$ missense mutation was not associated with MODY diabetes, it does seem to be linked to susceptibility to type 1 diabetes, as discussed below. A recent study reported a linkage between a homozygous missense mutation $(R 121 W)$ in PAX4 and type 2 diabetes in a Japanese population [6]. The mutant allele occurred at a low frequency $(2.0 \%)$ within this population [6], and Kanatsuka and coworkers described the same PAX4 mutation in patients with late-onset type 2 diabetes [7]. Interestingly, this mutation was associated with impaired first-phase insulin secretion, providing further evidence for the involvement of PAX4 in beta cell function [7]. However, it is not clear from these studies whether the R121W PAX4 missense mutation, whose functional effects have not been demonstrated in insulinproducing cells, may affect beta cell mass in these patients.

What is the situation in type 1 diabetes? Through microsatellite analysis of Scandinavian multiplex families, Holm and co-workers demonstrated a linkage between type 1 diabetes and a region on chromosome 7 (7q32) that carries PAX4, but they were unable to identify variation within the coding region of the gene [8]. There is thus evidence that variations in $P A X 4$ could be associated with susceptibility to type 1 diabetes, but the nature of this $P A X 4$ variation and its functional consequences are yet to be established.

The paper by Biason-Lauber and coworkers in this issue reports on the identification of PAX4 variants within the coding region of the gene and their functional effects in insulin-producing cells [3]. The authors screened 1,468 individuals for mutations in PAX4 through allelic discrimination and sequence analysis. Subjects included 379 schoolchildren with type 1 diabetes, 1,070 non-diabetic controls, and 19 schoolchildren who tested positive for anti-islet cell antibodies, but did not develop type 1 diabetes. A single nucleotide polymorphism, $A 1168 C$, was identified in exon 9 of $P A X 4$. The $A$ variant corresponded to a proline residue at position 321 of the protein, and the $C$ variant coded for histidine at this position. It should be noted that this is the PAX4 variant that could not be linked to MODY diabetes in French families [5]. In the study of Biason-Lauber 
et al., 77\% (Swiss cohort) and 72\% (German cohort) of the schoolchildren with type 1 diabetes carried the $C / C$ genotype, whereas only $30 \%$ of the control subjects and $10 \%$ of the antibody-positive non-diabetic children were $C / C$ carriers. The risk of developing type 1 diabetes was calculated to be 3.75-fold higher for individuals carrying the $C / C$ genotype than for individuals carrying the $A / C$ or $A / A$ genotype. It is of particular interest that antibody-positive children with the $A / C$ genotype had a significantly lower risk of developing manifest type 1 diabetes than those with other genotypes.

The authors next investigated the involvement of PAX4 variants in the development and function of endocrine cells. Human PAX4 $C$ and PAX4 $A$ variants were overexpressed in cell lines with a beta cell phenotype ( $\beta \mathrm{TC} 3)$, an alpha cell phenotype ( $\alpha \mathrm{TC} 1.9)$ and a non-endocrine phenotype (NIH3T3) [3]. Compared with PAX4A and the combination of the two variants, PAX $4 C$ repressed the transcription of a proinsulin promoter reporter construct to a lesser degree than PAX $4 A$ and the combination of the two variants, and showed impaired binding to DNA target sequences in electrophoretic mobility shift assays. Glucose-dependent cell proliferation was lower in $\beta \mathrm{TC} 3$ cells, but not in $\alpha \mathrm{TC} 1.9$ or NIH3T3 cells, after overexpression of the PAX4C variant. From these data, PAX4C could be interpreted as a 'diabetic' variant that limits the proliferation of beta cells in response to glucose stimulation. It should, however, be noted that these results were obtained using permanent cell lines. One of the crucial characteristics of these cell lines is their immortalisation, which results in constitutive cell growth. Consequently the results do not necessarily reflect the situation in primary beta cells, either during development or regeneration in the adult state. It would be useful to prove the importance of PAX4C for cell function and regeneration in primary beta cells, e.g. by using viral vector systems and by generating transgenic mice. These studies would help to verify PAX4C as a 'diabetic' variant of this homeobox transcription factor.

What are the implications of these data for experimental and clinical type 1 diabetes? First, they should focus our attention on defects in beta cell regeneration pathways in diabetic individuals and animal models of type 1 diabetes. The regenerative potential of the endocrine pancreas is generally accepted, but it remains unclear whether defects in regenerative capacity really exist in diabetic patients or in animal models. It therefore remains to be determined whether these defects are crucial for susceptibility to the development of type 1 diabetes. Second, genes encoding proteins involved in beta cell differentiation and regeneration could serve as prognostic markers, either for suscep- tibility to type 1 diabetes or for progression of the disease. Biason-Lauber and coworkers showed that antibody-positive children carrying the $A / C$ genotype of the $A 1168 \mathrm{C}$ single nucleotide polymorphism in PAX4 did not develop a diabetic syndrome [3]. The authors speculate that an improved regenerative potential might counterbalance the autoimmune destruction of beta cells, and this plausible hypothesis should be verified by prospective genetic studies on larger cohorts of antibody-positive children. Third, as an ultimate goal, an understanding of defects in pathways affecting the regenerative capacity of beta cells may open the door for new therapeutic interventions. This does not necessarily mean the correction of 'diabetic' variants of a gene by the use of viral vectors or physical gene transfer. In the era of modern drug design, compounds that increase the activity of the PAX $4 C$ variant could be developed in order to restore the regenerative capacity of beta cells. BiasonLauber and coworkers have elegantly shown that defective beta cell regeneration may be an essential and-so farunderestimated component of the pathogenesis of autoimmune diabetes [3]. It would be worthwhile to employ this experimental strategy to study other genes involved in beta cell differentiation to gain an insight into the role of regeneration in susceptibility to type 1 diabetes.

\section{References}

1. Eizirik DL, Mandrup-Poulsen T (2001) A choice of death - the signal-transduction of immune-mediated beta-cell apoptosis. Diabetologia 44:2115-2133

2. Homo-Delarche F, Drexhage HA (2004) Immune cells, pancreas development, regeneration and type 1 diabetes. Trends Immunol 25:222-229

3. Biason-Lauber A, Boehm B, Lang-Muritano M et al (2005) Association of childhood type 1 diabetes mellitus with a variant of PAX4: possible link to beta cell regenerative capacity. Diabetologia 48:DOI 10.1007/s00125-005-1723-5

4. Sosa-Pineda B (2004) The gene Pax4 is an essential regulator of pancreatic beta-cell development. Mol Cells 18:289-294

5. Dupont S, Vionnet N, Chevre JC et al (1999) No evidence of linkage or diabetes-associated mutations in the transcription factors BETA2/NEUROD1 and PAX4 in Type II diabetes in France. Diabetologia 42:480-484

6. Shimajiri Y, Sanke T, Furuta H et al (2001) A missense mutation of Pax 4 gene $(\mathrm{R} 121 \mathrm{~W})$ is associated with type 2 diabetes in Japanese. Diabetes 50:2864-2869

7. Kanatsuka A, Tokuyama Y, Nozaki O, Matsui K, Egashira T (2002) Beta-cell dysfunction in late-onset diabetic subjects carrying homozygous mutation in transcription factors NeuroD1 and Pax4. Metabolism 51:1161-1165

8. Holm P, Rydlander B, Luthman H, Kockum I (2004) Interaction and association analysis of a type 1 diabetes susceptibility locus on chromosome 5q11-q13 and the 7q32 chromosomal region in Scandinavian families. Diabetes 53:1584-1591 